Arch. Tierz., Dummerstorf 46 (2003) 3, 257-267

Aus der Thüringer Landesanstalt für Landwirtschaft Jena, Abteilungen Tierproduktion ${ }^{1}$ und Untersuchungswesen ${ }^{2}$

WERNER REICHARDT ${ }^{1}$, ERHARD GERNAND ${ }^{1}$, SIMONE MÜLLER ${ }^{1}$, HORST HARTUNG ${ }^{2}$, BÄRBEL ECKERT ${ }^{2}$ und UTA BRAUN ${ }^{1}$

\title{
Erhebungen zur Fettsäurenzusammensetzung von Rückenfett bei Thüringer Schweinen sowie zum Fett von Thüringer Knackwürsten aus dem Einzelhandel
}

\author{
Herrn Professor Dr. Dr. h. c. Klaus Ender zum 60. Geburtstag gewidmet
}

\begin{abstract}
Title of the paper: Examinations to the fatty acid composition of backfat at Thuringian pigs as well as to the fat of Thuringian cracking sausages from the retail trade

The fat from the outer back bacon of Thuringian pigs $(n=751)$ was examined several years with regard to their fatty acid composition. The content of polyunsaturated fatty acids (PUFA) ranged at the backfat from 7.3 to $12.7 \%$ and the relationship of the $\mathrm{n}-6$ and $\mathrm{n}-3$ fatty acids varied from 4.4 to 11.3 . PUFA contents in the backfat $>$ $12 \%$ were explained by high $\mathrm{C} 18: 2$ or $\mathrm{C} 18: 3$ contents of the food.

Examination of the fatty acid composition of the fat of 21 fresh cracking sausages from the Thuringian retail trade on a random basis yielded, that the contents of PUFA and linolenic acid ranged from 6.2 to $15.8 \%$ respectively from 0.8 to $2.0 \%$ as well as the quotient of n-6 to $n-3$ fatty acids varied from 3.6 to 10.3 .
\end{abstract}

Key Words: pig, backfat, sausage, fatty acid composition

\section{Zusammenfassung}

In einer mehrjährigen Erhebung wurde von Thüringer Schweinen das Fett aus dem äußeren Rückenspeck ( $\mathrm{n}=$ 751) hinsichtlich der Fettsäurenzusammensetzung untersucht. Der Polyenfettsäuren (PUFA)-Gehalt schwankte im Rückenfett zwischen 7,3 und 12,7 \%, während das Verhältnis der n-6- und n-3-Fettsäuren von 4,4 bis 11,3 variierte. PUFA-Gehaltswerte im Rückenfett $>12 \%$ waren auf zu hohe C18:2- oder C18:3-Anteile im eingesetzten Futter zurückzuführen. Die stichprobenartige Untersuchung der Fettsäurenzusammensetzung des Fettes von frischen 21 Knackwürsten aus dem Thüringer Einzelhandel ergab, dass der Gehalt an PUFA von 6,2$15,8 \%$, an Linolensäure von 0,8 bis 2,0 \% sowie der Quotient von n-6- zu n-3-Fettsäuren von 3,6 bis 10,3 schwankte.

Schlüsselwörter: Schwein, Rückenfett, Wurst, Fettsäurenmuster

1. Einleitung

Die Zusammensetzung tierischer Fette ist seit Jahren Gegenstand intensiver Forschung und zahlreicher Publikationen. Die diesbezüglichen Aspekte reichen von den Auswirkungen der Fettqualität auf die Qualität von Verarbeitungsprodukten (WARNANTS u.a., 1996, 1998; GLÄSER, 2000), die gesundheitlichen Auswirkungen der einzelnen Fettsäuren auf den Menschen (JAHREIS und BOCHMANN, 1998) bis hin zur Erhöhung des ernährungsphysiologischen Wertes tierischer Fette. KREUZER (1994), ENDER u.a. (1997) sowie SCHEEDER u.a. (2001a, b) wiesen auf zunehmende Zielkonflikte zwischen Beschaffenheitsanforderungen und diätetischer Qualität bei tierischen Fetten hin. Die Empfehlungen der Deutschen Gesellschaft für Ernährung (Refe- 
renzwerte 2000) bezüglich der menschlichen Ernährung umfassen folgende Aussagen zu den Nahrungsfetten:

- 25 - $30 \%$ der täglichen Energieaufnahme von 2.100 kcal sollte Fett sein

- die über Fett aufgenommene Energie sollte zu

- $10 \%$ aus gesättigten Fettsäuren (SFA)

- 8 bis $13 \%$ aus einfach ungesättigten Fettsäuren (Monoenfettsäuren, MUFA)

- $7 \%$ aus mehrfach ungesättigten Fettsäuren (Polyenfettsäuren, PUFA) mit einem

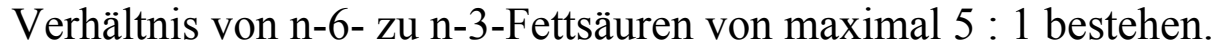

Zu den n-6-Polyenfettsäuren gehören die Linolsäure (C18:2; LA), die Eicosatriensäure (C20:3) und die Arachidonsäure (C20:4), während die $\alpha$-Linolensäure (C18:3; LNA; ALA), die Eicosapentaensäure (C20:5; EPA) sowie die Docosahexaensäure (C22:6; DHA) zu den n-3-Polyenfettsäuren rechnen. Beide Polyenfettsäurentypen unterscheiden sich in der Position der zur Methylgruppe endständigen Doppelbindung und stellen wichtige Bausteine menschlicher Zellmembranen dar oder dienen als Vorläufersubstanzen von Gewebshormonen. n-6-Fettsäuren wie C18:2 sind vor allem in Maiskeimöl, Sonnenblumenöl, Distelöl, Sojaöl und den daraus hergestellten Margarinesorten zu finden. C20:4 ist Bestandteil tierischer Lebensmittel. Weizenkeimöl, Leinsamenöl, Rapsöl (C18:3) und die Fette von Seefischen (C20:5, C22:6) enthalten hohe Anteile von n-3-Fettsäuren.

Bei der in Thüringen traditionsreichen Produktion von Dauerwurst spielt besonders die Fettqualität des Rückenspecks von Schweinen eine entscheidende Rolle. WENK und PRABUCKI (1990) schlugen für eine gute Fettqualität in der äußeren Rückenspeckschicht von Schweinen für die Schweiz folgende Grenzwerte vor:

- Summe aller ungesättigten Fettsäuren (UFA) $<59$ Mol-\%

- Summe aller Polyenfettsäuren (PUFA) $<12$ Mol- $\%$

- Summe aller Dienfettsäuren (z.B. C18:2) $<10$ Mol-\%

- Summe aller Trienfettsäuren (z.B. C18:3) $<1$ Mol-\%

- Summe aller Tetraenfettsäuren (z.B. C20:4) $<0,5$ Mol- $\%$

- Summe aller Pentaen- und Hexaenfettsäuren (z.B. C20:5, C22:6) < 1 Mol-\%

- Fettzahl (Iodzahl) $<58$

GLODEK (1997) forderte für deutsche Schweinezüchter zunächst eine umfassende Bestandsaufnahme der Fettsäurenzusammensetzung bei den Lipidgeweben von Schweinen als vordringliche Aufgabe für die staatliche Leistungsprüfung und für Forschungseinrichtungen. In diesem Sinne war es Absicht einer mehrjährigen Erhebung, stichprobenartige Aussagen zum Fettsäurenmuster des Rückenfetts von Thüringer Schweineherkünften zu gewinnen.

2. Material und Methoden

2.1. Tiere / Wurstproben

In dem Beitrag sind drei verschiedene Thüringer Zustandserhebungen zur Fettsäurenzusammensetzung tierischer Fette zusammengefasst worden:

- die Fettsäurenzusammensetzung des Fetts von Rückenspeck von Tieren aus der Leistungsprüfanstalt des Landes (LPA) und aus einem Markenfleischprogramm

- die Fettsäurenzusammensetzung des Fetts von Rückenspeck von Tieren von Direktvermarktern aus Thüringen 
- die Fettsäurenzusammensetzung des Wurstfetts von Thüringer Knackwürsten aus dem Handel oder von Direktvermarktern

Bei den untersuchten Schweinen handelte es sich um Sauen und Börge aus der Thüringer Leistungsprüfanstalt (LPA) sowie aus Thüringer Mastbetrieben. Das Tiermaterial besteht aus Reinzucht- und Kreuzungstieren der Mutterrassen: Deutsches Edelschwein (DE), Deutsche Landrasse (DL) und Leicoma (Lc); aus Prüftieren der Endstufenvarianten: Pietrain (Pi - Reinzucht), Duroc (Du - Reinzucht) und Hampshire x Pietrain (HaPi) sowie aus Masthybriden: Pi x DEDL, HaPi x DL, Du x DEDL. Die Wurstproben wurden zufallsmäßig über ganz Thüringen verteilt in Fleischereien, Läden von Direktvermarktern sowie Discountmärkten erworben. Die statistische Auswertung der erhobenen Daten erfolgte mit dem Programm SPSS 11.0 (multiple t-Tests mit versuchsbezogener Irrtumswahrscheinlichkeit von $\alpha=0,05$ nach Scheffé und nach Bonferroni).

\subsection{Extraktion von Fett aus Rückenspeck}

\subsubsection{Bei Partieuntersuchungen}

Die Proben entstammen der äußeren Rückenspeckschicht bei der Anschnittstelle des Schlachtkörpers am 13./14. Brustwirbel, an der auch die Fleischprobe für die Analyse des intramuskulären Fettgehaltes entnommen wird. Der Rückenspeck ist einen Tag nach der Probenahme von der Schwarte befreit und weiter aufbereitet worden. Zunächst erstreckte sich die Extraktion des Fettes von Rückenspeck auf Mischproben. Hierzu wurden von 6 Tieren einer Prüfgruppe je 1 bis 3 g Speck eingewogen und miteinander in einem Homogenisator zerkleinert. Die Aufbewahrung der homogenisierten Speckproben erfolgte in Plastampullen bis zu vierzehn Tage bei $-18^{\circ} \mathrm{C} .1-1,3 \mathrm{~g}$ dieser Speckmischung sind nach dem Auftauen in einem $300 \mathrm{ml}$ Erlenmeyerkolben mit NS 29 mit $150 \mathrm{ml}$ einer Lösungsmittelmischung von Dichlormethan und Methanol im Verhältnis 9:1 (v/v) überschichtet worden und standen nach mehrfachem Umschütteln über Nacht. Am nächsten Morgen wurde das Lösungsmittelgemisch mittels Trichter und engporigem Faltenfilter in ein Kurzhalsstehkolben abfiltriert und unter Vakuum abdestilliert. Das zurückbleibende Speckfett ist anschließend in einer Gefriertrocknungsanlage im Vakuum bei $40{ }^{\circ} \mathrm{C}$ schonend nachgetrocknet, in $5 \mathrm{ml}$ Plastampullen mit luftdichtem Verschluss abgefüllt und bis zur Analyse bei $-18^{\circ} \mathrm{C}$ aufbewahrt worden. Das Auftauen der Proben erfolgte 1Tag vor der Untersuchung (Iodzahl) in einem Kühlschrank bei $4^{\circ} \mathrm{C}$.

\subsubsection{Bei Einzeluntersuchungen}

Die Entnahme und Aufbereitung des Rückenspecks entsprachen den Angaben unter 2.3.1. Von den Speckproben wurde jeweils nur die Anzahl aufgetaut, die am gleichen Tag extrahiert und gaschromatographisch analysiert werden konnte. Im Unterschied zur Extraktion der Mischproben sind 0,5 g homogenisierter Speck in einem $15 \mathrm{ml}-$ Schliffröhrchen (NS 14,5) mit $5 \mathrm{ml} \mathrm{n-Hexan/Aceton-Gemisch}(2: 1, \mathrm{v} / \mathrm{v})$ versetzt und danach mit Hilfe eines Glasstabs in der Extraktionslösung locker aufgerührt worden. Die Schliffröhrchen wurden 1 Stunde mit Ultraschallbad (Bandelin TK 30: $50 \mathrm{KHz} /$ 144 W) behandelt. Nach kurzem Zentrifugieren konnte die flüssige Phase in ein weiteres 15 ml-Schliffröhrchen abdekantiert werden. Das Lösungsmittelgemisch ist im Wasserstrahlpumpenvakuum entfernt worden. Es erfolgte eine zweistündige Nach- 
trocknung des extrahierten Speckfetts bei $40^{\circ} \mathrm{C}$. Bei einem Vergleich verschiedener Lösungsmittelmischungen (REICHARDT und ECKERT, 2003) erzielte diese Extraktionsvariante die höchste Iodzahl nach WIJS sowie die beste Korrelation derselben zur gaschromatographisch ermittelten Iodzahl $(r=0,84)$.

\subsection{Die Extraktion von Rohfett aus Futtermittelproben}

Die Extraktion der Fette aus den gemahlenen Prüffuttermischungen oder Futterpellets erfolgte im Originalzustand ohne vorherigen Säureaufschluss. Teilweise mussten die Proben mittels Homogenisator weiter zerkleinert werden. $10 \mathrm{~g}$ Einwaage sind mit $3 \mathrm{~g}$ wasserfreiem Natriumsulfat vermischt und mit $200 \mathrm{ml}$ Petrolether (Siedebereich 40-60 ${ }^{\circ}$ C) 6,5 Stunden nach dem Soxhlet-Verfahren extrahiert worden.

\subsection{Extraktion von Fett bei Wurst}

$10 \mathrm{~g}$ homogenisierte Wurst sind mit $100 \mathrm{ml}$ n-Hexan im SOXTEC Avanti 2050 behandelt worden (Siedezeit: 30 Min., Extraktionszeit: 45 Min., Destillationszeit: 15 Min., Nachtrocknung: 5 Min.). Das extrahierte Fett wurde in luftdichte Plastampullen überführt und bis zur gaschromatographischen Analyse bei $-18^{\circ} \mathrm{C}$ aufbewahrt.

\subsection{Die Bestimmung der Iod- oder Fettzahl}

Die Iodzahl, auch als Fettzahl bezeichnet, ist eine methodisch definierte Maßzahl für den Anteil an ungesättigten Fettsäuren in Fetten und Ölen. Die Bestimmung der Iodzahl (IZ) erfolgte nach der Methode von WIJS mittels Iodtrichlorid (IUPAC, 1992). Hierzu sind 300 - $350 \mathrm{mg}$ des Fettes in einen $500 \mathrm{ml}$ Erlenmeyerkolben NS 29 genau eingewogen worden. Zur Richtigkeitskontrolle enthielten alle Untersuchungsserien stets gleichzeitig Proben von Oliven- $(I Z=84)$, Sonnenblumen- $(I Z=132)$ und Leinöl $(I Z=186)$. Die Sollwertangaben für die Iodzahlen dieser Öle entstammen dem Lehrbuch von BELITZ und GROSCH (1992).

\subsection{Die gaschromatographische Bestimmung der Fettsäurenzusammen- setzung}

Zur gaschromatographischen Bestimmung wurden $100 \mathrm{mg}$ extrahiertes Fett in 2,5 ml tertiär-Butyl-Methylether gelöst. $100 \mu 1$ dieser Lösung werden zur Umesterung mit $100 \mu 1$ einer Lösung von Trimethylsulfoniumhydroxid in Methanol versetzt. Die Analyse der Fettsäurenzusammensetzung erfolgte mittels Kapillargaschromatographie (Gaschromatograph VARIAN STAR 3400; DB-WAX-Säule $30 \mathrm{~m}$; Ø 0,32 mm; $1 \mu 1$ Injektionsvolumen) als Doppelbestimmung. Die Auswertung beruht auf der Methode der externen Kalibration unter Nutzung von C15:0 als innerem Standard. In die Auswertung sind die Fettsäuren C12:0, C14:0, C15:1, C16:0, C16:1, C17:0, C17:1, C18:0, $\mathrm{C} 18: 1$ (alle Isomere), C18:2 (alle Isomere), C18:3, C20:0, C20:4 und C22:0 einbezogen worden. C20:4 wurde nur zeitweise bei der Analyse berücksichtigt. Die Angaben zu den Fettsäurenanteilen beziehen sich generell auf Masse-\% Fettsäurenmethylester.

3. Ergebnisse

3.1. Das Fettsäurenmuster von Rückenfett und von Futter

Die Ergebnisse der gaschromatographischen Analyse des Fettsäurenmusters und der Iodzahl-Bestimmung bei Rückenfett von Schweinen aus der Thüringer LPA sind in 
Tabelle 1 zusammengefasst. Analog zur Verfahrensweise in Schweizer Schlachthöfen (vgl. SCHEEDER u.a., 1999) wurde bei den Tieren des Prüfjahrs 1995 die Iodzahl des Fettes von partieweise extrahiertem Rückenspeck bestimmt. Die partieweise Untersuchung der Iodzahl von Rückenspeck gestattet keine Aussage über das Einzeltier. Daher erfolgte 1996 und 1997 die Iodzahlbestimmung nicht mehr an Mischproben von Rückenfett, sondern an Einzelproben.

Tabelle 1

Fettsäurenzusammensetzung und Iodzahl von Rückenfett bei Schweinen aus der Thüringer Leistungsprüfanstalt (LPA) sowie aus einem Markenfleischprogramm (Einzelproben; Masse-\%) (Fatty acid composition and iodine value of backfat at pigs from the Thuringian performance testing station (LPA) as well as from a label meat production (singles samples; mass \%))

\begin{tabular}{|c|c|c|c|c|c|c|c|c|}
\hline \multirow[b]{2}{*}{ Merkmal } & \multicolumn{2}{|c|}{$\begin{array}{l}\text { LPA } 1995 \\
(\mathrm{n}=378)\end{array}$} & \multicolumn{2}{|c|}{$\begin{array}{l}\text { LPA } 1996 \\
(\mathrm{n}=130)\end{array}$} & \multicolumn{2}{|c|}{$\begin{array}{l}\text { Markenfleisch- } \\
\text { programm } 1997 \\
(\mathrm{n}=60)\end{array}$} & \multicolumn{2}{|c|}{$\begin{array}{l}\text { LPA } 1999 / 2000 \\
\quad(n=125)\end{array}$} \\
\hline & $\varnothing$ & $\mathrm{S}$ & $\varnothing$ & $\mathrm{s}$ & $\varnothing$ & $\mathrm{S}$ & $\varnothing$ & $\mathrm{S}$ \\
\hline C14:0 & $1,67^{b}$ & 0,32 & $1,53^{\mathrm{a}}$ & 0,28 & $1,83^{\mathrm{c}}$ & 0,15 & $1,75^{b}$ & 0,14 \\
\hline C16:0 & $24,88^{\mathrm{a}}$ & 1,52 & $28,21^{b}$ & 3,43 & $30,35^{\mathrm{c}}$ & 0,95 & $29,75^{\mathrm{c}}$ & 1,09 \\
\hline C16:1 & $2,28^{\mathrm{a}}$ & 0,31 & $2,32^{\mathrm{a}}$ & 0,65 & $3,84^{\mathrm{c}}$ & 0,46 & $3,43^{\mathrm{b}}$ & 0,45 \\
\hline C17:0 & $1,99^{\mathrm{c}}$ & 1,25 & $0,35^{\mathrm{a}}$ & 0,08 & $1,12^{b}$ & 0,16 & $0,89^{b}$ & 0,23 \\
\hline C18:0 & $12,72^{\mathrm{a}}$ & 1,48 & $17,89^{\mathrm{c}}$ & 3,68 & $16,58^{b}$ & 0,89 & $16,61^{b}$ & 1,46 \\
\hline C18:1 & $37,00^{\mathrm{c}}$ & 2,80 & $34,05^{b}$ & 4,24 & $35,03^{b}$ & 1,40 & $32,30^{\mathrm{a}}$ & 1,25 \\
\hline C18:2 & $11,51^{\mathrm{c}}$ & 1,99 & $10,95^{\mathrm{c}}$ & 2,37 & $6,34^{\mathrm{a}}$ & 0,86 & $10,25^{b}$ & 1,15 \\
\hline C18:3 & $1,04^{b}$ & 0,19 & $1,17^{\mathrm{c}}$ & 0,25 & $0,91^{\mathrm{a}}$ & 0,15 & $1,46^{\mathrm{d}}$ & 0,16 \\
\hline C20:0 & n. b. & - & $0,30^{\mathrm{b}}$ & 0,33 & $0,22^{\mathrm{a}}$ & 0,04 & $0,41^{\mathrm{c}}$ & 0,06 \\
\hline C20:4 & $0,19^{\mathrm{a}}$ & 0,07 & $0,16^{\mathrm{a}}$ & 0,25 & n. b. & - & n. b. & - \\
\hline SFA & $45,32^{a}$ & 3,14 & $50,35^{\mathrm{b}}$ & 6,62 & $53,45^{\mathrm{c}}$ & 1,39 & $52,13^{c}$ & 2,34 \\
\hline UFA & $52,02^{\mathrm{c}}$ & 3,22 & $48,96^{\mathrm{b}}$ & 5,71 & $46,55^{\mathrm{a}}$ & 1,40 & $47,92^{b}$ & 2,18 \\
\hline MUFA & 39,28 a & 2,71 & $36,69^{b}$ & 3,91 & $39,30^{\mathrm{a}}$ & 1,46 & $36,21^{b}$ & 1,42 \\
\hline PUFA & $12,74^{\mathrm{c}}$ & 2,16 & $12,27^{\mathrm{c}}$ & 2,62 & $7,25^{\mathrm{a}}$ & 0,99 & $11,71^{\mathrm{b}}$ & 1,29 \\
\hline PUFA/SFA & $0,28^{\mathrm{d}}$ & 0,06 & $0,25^{\mathrm{c}}$ & 0,09 & $0,14^{\mathrm{a}}$ & 0,02 & $0,23^{\mathrm{b}}$ & 0,03 \\
\hline $\mathrm{n}-6 / \mathrm{n}-3 *)$ & $11,35^{\mathrm{c}}$ & 0,70 & $9,82^{\mathrm{b}}$ & 2,58 & $7,07^{\mathrm{a}}$ & 0,92 & $7,02^{\mathrm{a}}$ & 0,36 \\
\hline Iodzahl & $68,13^{b}$ & 2,23 & $68,54^{b}$ & 3,94 & $58,57^{\mathrm{a}}$ & 2,90 & n. b. & - \\
\hline
\end{tabular}

*) nur C18:2 / C18:3 berücksichtigt; $\varnothing=$ Mittelwert; $\mathrm{s}=$ Standardabweichung; $\mathrm{n}$. b. = nicht bestimmt;

Werte mit dem gleichen Hochbuchstaben unterscheiden sich nicht signifikant $(\alpha=0,05)$;

Überschreitungen der Schweizer Grenzwerte für gute Fettqualität im Rückenspeck wurden fett gedruckt; Eine Umrechnung dieser Grenzwerte auf der Basis des Fettsäurenmusters von Tabelle 1 ergibt, dass der Unterschied zwischen Mol- $\%$ und Masse- $\%$ vernachlässigt werden kann: 10 Mol-\%= 10,33 Masse-\% bei C18:2; 1 Mol-\%=1,03 Masse- $\%$ bei C18:3; 0,5 Mol- $\%=0,56$ Masse- $\%$ bei C20:4

Tabelle 2

Fettsäurenzusammensetzung der Futterpellets in der Thüringer LPA - 1995 (Masse-\%; n = 7 verschiedene Proben) (Fatty acid composition of the feeding pellets in the Thuringian performance testing station - 1995 (mass \%; $\mathrm{n}=7$ different samples))

\begin{tabular}{l|rl|l|cc}
\hline Merkmal & \multicolumn{1}{|c|}{$\varnothing$} & s & Merkmal & $\varnothing$ & s \\
\hline C14:0 & 2,74 & 0,37 & C18:3 & 3,54 & 0,48 \\
C16:0 & 20,64 & 0,87 & C20:0 & 0,23 & 0,06 \\
C16:1 & 2,21 & 0,29 & C20:4 & 34,89 & 0,06 \\
C17:0 & 4,49 & 1,41 & SFA & 60,50 & 2,55 \\
C18:0 & 3,95 & 0,69 & UFA & 22,46 & 3,75 \\
C18:1 & 20,25 & 2,45 & MUFA & 38,74 & 2,72 \\
C18:2 & 35,47 & 2,71 & PUFA & 3,11 \\
\hline
\end{tabular}

Die 1995 gefundene Zusammensetzung des Rückenfetts führte dazu, auch das Fettsäurenmuster von sieben Futterchargen zu untersuchen (Tab. 2), die in diesem Jahr in der LPA für die Schweinefütterung eingesetzt wurden. Die Anteile an Linol- und Linolensäure im Futter waren hoch. Unter Berücksichtigung der Tatsache, dass die Fettsäuren zu etwa $90 \%$ zur Molmasse des Triacylglyceridmoleküls beitragen, lässt sich bei ei- 
nem mittleren Rohfettgehalt von $4 \%$ errechnen, dass 1 kg Futterpellets im Durchschnitt etwa $14 \mathrm{~g}$ Polyensäuren enthalten haben.

Bei Direktvermarktungsunternehmen in Thüringen auftretende Probleme mit der Haltbarkeit von selbst hergestellter Dauerwurst gaben den Anlass, Untersuchungen zur Fettsäurenzusammensetzung von Rückenspeck ihrer Mastschweine (Tab. 3) sowie des Futters (Tab. 4) vorzunehmen. Die Betriebe mästeten Absetzer der gleichen Herkunft (Börge aus Rotationskreuzung) und setzten das gleiche Futter ein.

Tabelle 3

Fettsäurenzusammensetzung bei Rückenfett der Börge von Thüringer Direktvermarktern (Masse-\%; Rotationskreuzung) (Fatty acid composition at backfat of barrows of Thuringian farms which commercialize directly (mass- $\%$; rotation crosses))

\begin{tabular}{c|c|c|c|c|c|c}
\hline Erhebungsjahr 1999 & \multicolumn{2}{|c|}{ Betrieb 1 } & \multicolumn{2}{c|}{ Betrieb 2 } & \multicolumn{2}{c}{ Betrieb 3 } \\
\hline $\mathrm{n}$ & \multicolumn{2}{|c|}{19} & \multicolumn{2}{c}{18} & \multicolumn{2}{c}{21} \\
\hline Merkmal & $\varnothing$ & $\mathrm{s}$ & $\varnothing$ & $\mathrm{s}$ & $\varnothing$ & $\mathrm{s}$ \\
\hline Schlachtmasse in kg & 142,4 & 7,6 & 90,5 & 13,9 & 144,2 & 20,0 \\
\hline C14:0 & $1,73^{\mathrm{a}}$ & 0,10 & $1,74^{\mathrm{a}}$ & 0,11 & $1,83^{\mathrm{b}}$ & 0,11 \\
$\mathrm{C} 16: 0$ & $29,96^{\mathrm{b}}$ & 0,87 & $28,87^{\mathrm{a}}$ & 0,90 & $29,14^{\mathrm{a}}$ & 0,63 \\
$\mathrm{C} 16: 1$ & $2,94^{\mathrm{a}}$ & 0,33 & $3,17^{\mathrm{a}}$ & 0,43 & $3,17^{\mathrm{a}}$ & 0,39 \\
$\mathrm{C} 17: 0$ & $0,97^{\mathrm{a}}$ & 0,18 & $0,95^{\mathrm{a}}$ & 0,16 & $0,92^{\mathrm{a}}$ & 0,38 \\
$\mathrm{C} 18: 0$ & $17,21^{\mathrm{b}}$ & 1,09 & $15,64^{\mathrm{a}}$ & 1,15 & $15,64^{\mathrm{a}}$ & 0,98 \\
C18:1 & $32,39^{\mathrm{a}}$ & 1,23 & $34,79^{\mathrm{b}}$ & 1,68 & $35,92^{\mathrm{c}}$ & 1,22 \\
C18:2 & $9,98^{\mathrm{c}}$ & 0,67 & $9,11^{\mathrm{b}}$ & 1,08 & $8,33^{\mathrm{a}}$ & 0,52 \\
C18:3 & $\mathbf{1 , 5 4}^{\mathrm{a}}$ & 0,14 & $2, \mathbf{0 5} 5^{\mathrm{b}}$ & 0,18 & $\mathbf{1 , 4 6}^{\mathrm{a}}$ & 0,18 \\
C20:0 & $0,34^{\mathrm{a}}$ & 0,04 & $0,40^{\mathrm{b}}$ & 0,05 & $0,38^{\mathrm{b}}$ & 0,04 \\
SFA & $52,68^{\mathrm{b}}$ & 1,46 & $50,34^{\mathrm{a}}$ & 1,64 & $50,64^{\mathrm{a}}$ & 1,39 \\
UFA & $47,35^{\mathrm{a}}$ & 1,46 & $49,77^{\mathrm{b}}$ & 1,71 & $49,36^{\mathrm{b}}$ & 1,43 \\
MUFA & $35,82^{\mathrm{a}}$ & 1,28 & $38,48^{\mathrm{b}}$ & 1,84 & $39,58^{\mathrm{c}}$ & 1,29 \\
PUFA & $11,52^{\mathrm{b}}$ & 0,80 & $11,16^{\mathrm{b}}$ & 1,24 & $9,79^{\mathrm{a}}$ & 0,66 \\
PUFA / SFA & $0,22^{\mathrm{b}}$ & 0,02 & $0,22^{\mathrm{b}}$ & 0,03 & $0,19^{\mathrm{a}}$ & 0,02 \\
n-6 / n-3 & $6,50^{\mathrm{c}}$ & 0,38 & $4,44^{\mathrm{a}}$ & 0,31 & $5,75^{\mathrm{b}}$ & 0,50 \\
\hline
\end{tabular}

Überschreitungen der Schweizer Grenzwerte für gute Fettqualität im Rückenspeck wurden fett gedruckt; Werte mit dem gleichen Hochbuchstaben unterscheiden sich nicht signifikant $(\alpha=0,05)$

Tabelle 4

Fettsäurenzusammensetzung des in den Betrieben 1 bis 3 eingesetzten Futters (Masse-\%; Vierfachbestimmung) (Fatty acid composition of the feed which was used in the farms 1 to 3 (mass-\%; quadruple determination))

\begin{tabular}{l|c|c|l|c|c}
\hline Merkmal & $\begin{array}{l}\text { unhomogenisiertes } \\
\text { Futter - } \varnothing\end{array}$ & $\begin{array}{l}\text { homogensiertes } \\
\text { Futter }-\varnothing\end{array}$ & $\begin{array}{l}\text { Merk- } \\
\text { mal }\end{array}$ & $\begin{array}{l}\text { unhomogenisiertes } \\
\text { Futter - } ~\end{array}$ & $\begin{array}{l}\text { homogensiertes } \\
\text { Futter - }\end{array}$ \\
\hline C14:0 & 1,25 & 1,10 & C18:3 & 5,39 & 5,92 \\
C16:0 & 25,19 & 24,58 & C20:0 & 0,34 & 0,37 \\
C16:1 & 2,10 & 1,87 & C22:0 & 0,30 & 0,34 \\
C17:0 & 1,27 & 1,41 & SFA & 39,66 & 38,09 \\
C18:0 & 9,78 & 8,69 & UFA & 60,33 & 61,92 \\
C18:1 & 24,29 & 22,97 & MUFA & 26,89 & 25,32 \\
C18:2 & 28,08 & 30,68 & PUFA & 33,44 & 36,60 \\
\hline
\end{tabular}

- Fettgehalt der Futterpellets (Vierfachbestimmung): unhomogenisiert: 2,08 \%; homogenisiert: 2,48\%

- PUFA-Gehalt(homogenisierte Probe): $24,8 \mathrm{~g}$ Fett $/ \mathrm{kg}$ x 0,9 x 36,6 \% PUFA / $100 \%=8,2 \mathrm{~g} / \mathrm{kg}$ Futter

\subsection{Die Fettsäurenzusammensetzung von Wurstfett}

Zur Herstellung von bestimmten Dauerwürsten wird neben Muskelfleisch auch der Rückenspeck von Schweinen verwendet. Daher lag es nahe zu prüfen, welche Fettsäurenanteile den Verbraucher in Thüringen beim Verzehr solcher Würste erreichen. Tabelle 5 gibt die Zusammensetzung des Fettes von 2001 und 2002 erworbenen Würsten im Vergleich zu Sonnenblumenölmargarine, Rapsöl und Schweinefett wider. 
Tabelle 5

Fettsäurenzusammensetzung (Masse-\%) bei frischen Thüringer Knackwürsten (Fatty acid composition (mass \%) of fresh Thuringian cracking sausages)

\begin{tabular}{|c|c|c|c|c|c|c|c|c|c|}
\hline Probe / Merkmal & $\mathrm{C} 16: 0$ & $\mathrm{C} 16: 1$ & $\mathrm{C} 18: 0$ & $\mathrm{C} 18: 1$ & $\mathrm{C} 18: 2$ & $\mathrm{C} 18: 3$ & PUFA & UFA & $n-6 / n-3$ \\
\hline Knackwurst $1^{1)}$ & 25,33 & 2,93 & 11,83 & 38,92 & 14,35 & 1,40 & 15,75 & 57,9 & 10,3 \\
\hline Knackwurst $2^{2)}$ & 28,90 & 3,98 & 14,84 & 31,62 & 12,01 & 2,00 & 14,01 & 50,3 & 6,0 \\
\hline Knackwurst 3 & 30,30 & 3,62 & 17,39 & 33,25 & 8,83 & 1,33 & 10,16 & 47,4 & 6,6 \\
\hline Knackwurst 4 & 29,48 & 3,79 & 15,70 & 34,03 & 9,58 & 1,53 & 11,11 & 49,3 & 6,3 \\
\hline Knackwurst 5 & 28,96 & 3,68 & 15,64 & 33,75 & 9,77 & 1,56 & 11,33 & 49,1 & 6,3 \\
\hline Knackwurst 6 & 29.73 & 4,76 & 14,90 & 34,75 & 8,55 & 1,45 & 10,00 & 48,9 & 5,5 \\
\hline Knackwurst $7^{3)}$ & 31,80 & 4,71 & 16,52 & 33,65 & 6,08 & 0,77 & 6,85 & 46,2 & 7,9 \\
\hline Knackwurst 8 & 31,18 & 3,83 & 16,70 & 31,70 & 7,90 & 1,54 & 9,44 & 45,5 & 5,1 \\
\hline Knackwurst 9 & 30,19 & 4,04 & 17,12 & 32,49 & 8,03 & 1,16 & 9,19 & 46,3 & 6,9 \\
\hline Knackwurst 11 & 29,99 & 3,84 & 15,96 & 34,52 & 7,53 & 1,03 & 8,56 & 47,4 & 7,3 \\
\hline Knackwurst 12 & 27,48 & 3,65 & 14,39 & 39,37 & 6,67 & 1,84 & 8,51 & 52,6 & 3,6 \\
\hline Knackwurst 13 & 29,67 & 4,03 & 14,97 & 38,16 & 5,41 & 0,79 & 6,20 & 49,0 & 6,8 \\
\hline Knackwurst 14 & 28,67 & 3,53 & 15,58 & 38,35 & 6,05 & 1,00 & 7,05 & 49,5 & 6,1 \\
\hline Knackwurst 15 & 29,28 & 4,61 & 15,62 & 35,40 & 6,99 & 1,32 & 8,31 & 49,0 & 5,3 \\
\hline Knackwurst 16 & 30,16 & 4,16 & 15,88 & 35,36 & 6,96 & 1,06 & 8,02 & 48,1 & 6,6 \\
\hline Knackwurst $17^{\text {4) }}$ & 30,40 & 3,89 & 16,05 & 35,88 & 5,94 & 1,07 & 7,01 & 47,4 & 5,6 \\
\hline Knackwurst 18 & 29,25 & 3,28 & 16,97 & 35,77 & 6,99 & 0,90 & 7,89 & 47,2 & 7,8 \\
\hline Knackwurst $20^{5)}$ & 30,21 & 3,43 & 16,23 & 31,99 & 8,58 & 1,15 & 9,73 & 45,6 & 7,5 \\
\hline Knackwurst 21 & 30,07 & 4,01 & 14,22 & 33,13 & 8,24 & 1,31 & 9,55 & 47,3 & 6,3 \\
\hline Knackwurst 22 & 30,68 & 3,89 & 16,13 & 33,17 & 8,24 & 1,23 & 9,47 & 47,1 & 6,7 \\
\hline Knackwurst 24 & 28,49 & 4,11 & 12,84 & 40,72 & 8,51 & 1,81 & 10,32 & 55,8 & 4,7 \\
\hline Knackwurst 25 & 30,43 & 3,98 & 15,60 & 34,75 & 10,05 & 1,41 & 11,46 & 50,9 & 7,1 \\
\hline $\begin{array}{c}\varnothing(\mathrm{n}=21, \text { ohne } \\
\text { Wurst } 7)\end{array}$ & 29,47 & 3,86 & 15,46 & 35,10 & 8,34 & 1,33 & 9,67 & 49,1 & 6,4 \\
\hline $\mathrm{s}$ & 1,28 & 0,40 & 1,34 & 2,65 & 2,07 & 0,32 & 2,25 & 3,11 & 1,3 \\
\hline \begin{tabular}{ll}
\multicolumn{2}{l}{ Ungarische } \\
Salami $\quad 10$
\end{tabular} & 30,61 & 3,98 & 15,82 & 35,06 & 7,65 & 0,61 & 8,26 & 47,8 & 12,5 \\
\hline $\begin{array}{l}\text { Französische } \\
\text { Salami } \quad 19^{6)}\end{array}$ & 29,84 & 2,46 & 16,83 & 32,66 & 10,15 & 0,99 & 11,14 & 46,6 & 10,3 \\
\hline $\begin{array}{ll}\text { Spanische } & \\
\text { Salami } & 23\end{array}$ & 29,80 & 3,51 & 15,37 & 33,04 & 10,33 & 1,11 & 11,44 & 48,5 & 9,3 \\
\hline $\begin{array}{l}\text { Sonnenblumen- } \\
\text { ölmargarine }^{7)}\end{array}$ & 8,09 & 0,16 & 11,30 & 43,78 & 34,20 & 0,32 & 34,52 & 78,5 & 106,9 \\
\hline 00-Rapsöl ${ }^{8)}$ & 6,5 & 1,0 & 1,4 & 58,0 & 10,5 & 14,2 & 24,7 & 83,7 & 0,7 \\
\hline Schweinefett ${ }^{9)}$ & 24,8 & 3,1 & 12,3 & 45,1 & 9,9 & 0,1 & 10,0 & 58,2 & 99,0 \\
\hline Rückenfett $^{10)}$ & 26,5 & 2,5 & 14,5 & 35,5 & 11,1 & 1,1 & 12,3 & 50,5 & 10,0 \\
\hline \multicolumn{10}{|c|}{$\begin{array}{l}\text { 1) Wurstfett war beim Anschnitt der Wurst bei Raumtemperatur ölig; } \\
\text { 2) Wurst war eine Woche nach dem Kauf und Anschnitt verdorben; } \\
\text { 3) geräucherte Wurst, die frische Wurst Nr. } 8 \text { entstammt der gleichen Produktionscharge; } \\
\text { 4) halb Schwein - halb Rind; } \\
\text { 5) } 20 \text { \% Anteil Rind; } \\
\text { () Wurst schmeckte eine Woche nach Anschnitt ranzig; } \\
\text { 7) nach PRECHT und MOLKENTIN (2000); } \\
\text { 8) nach DECKER u.a. (2000); } \\
\text { 9) nach GASSEN und HAMMES (1997); } \\
\text { 10) Rückenfett aus der Thüringer LPA: Prüfjahre 1995, 1996, 1999/2000 (vgl. Tabelle 1); } \mathrm{n}=633 \\
\text { Überschreitungen der Schweizer Grenzwerte für gute Fettqualität im Rückenspeck wurden fett gedruckt }\end{array}$} \\
\hline
\end{tabular}

Die in diesem Beitrag vorgestellten Ergebnisse sind das Resultat von Erhebungen und nicht von gezielten Versuchen. Aus den Daten der Tabelle 1 geht hervor, dass die Schweizer Grenzwerte für gute Fettqualität im Rückenspeck bei den Merkmalen C18:2 und C18:3 von den 1995, 1996 und 1999/2000 untersuchten Stichproben überschritten wird. Interessant ist der kontinuierliche Abfall der C18:2-Anteile bzw. Anstieg der C18:3-Anteile des Rückenfetts im Erhebungszeitraum. Die Stichprobe 1999/2000 unterschreitet sogar den PUFA-Grenzwert von $12 \%$ im Rückenfett. Mit 68,5 lag die Iod- 
zahl bei den 1996 durchgeführten Einzelanalysen auf dem Niveau der Partieanalysen von 1995, was belegt, dass mit der partieweisen Untersuchung der Iodzahl eine treffsichere schnelle Zustandsanalyse des Anteils von $\mathrm{C}=\mathrm{C}$-Doppelbindungen am Fett möglich ist. Die 1997 an Rückenfett von Schweinen aus einem Markenfleischprogramm ermittelten Daten zeichnen sich vor allem durch eine günstige durchschnittliche Iodzahl von 58,6 sowie durch relativ niedrige C18:2- und PUFA-Anteile aus. Diese Stichprobe überschritt im Mittelwert bei keinem Merkmal die Schweizer Grenzwerte für gute Fettqualität im Rückenfett.

Nach SCHWÖRER u.a. (1995) toleriert man beim Auszahlungspreis in Schweizer Schlachthöfen im Fett der äußersten Rückenspeckschicht maximal eine Fettzahl / Iodzahl von 62, um die technologische Verarbeitungseignung und oxidative Stabilität des Specks zu gewährleisten. Damit dieser Wert nicht überschritten wird, gaben PERDRIX und STOLL (1995) eine obere Grenze von 0,8 g Polyensäuren / MJ verdauliche Energie beim Schwein (ca. 12 g PUFA/kg Futter) vor. Anhand der Angaben von BODIS (1999) lässt sich errechnen, dass beim Fett des Rückenspecks von 360 Schweinen, die nach den Richtlinien des Zentralverbandes der deutschen Schweineproduktion e.V. (ZDS) für die Stationsprüfung auf Mastleistung, Schlachtkörperwert und Fleischbeschaffenheit gefüttert wurden (LPA Grub bzw. Versuchsstation Thalhausen), die der Iodzahl vergleichbare Fettzahl nur 57,3 betrug. Dieser Umstand und eine 2001 von MÜLLER u.a. (2003) durchgeführte Untersuchung zur Variation der Fettsäurenzusammensetzung des Prüffutters in den deutschen LPA provoziert Gedanken, zukünftig eine Standardisierung des Prüffutters in den LPA auch hinsichtlich des Fettsäurenmusters zu fordern. Die Fettsäurenzusammensetzung des Rückenfetts der Schweine aus der Thüringer LPA (Tab. 1) wurde auch der Varianzanalyse (Genkonstruktion, Geschlecht, Erhebungsjahr) sowie einer Varianzkomponentenschätzung unterworfen. Die Ergebnisse werden Gegenstand einer eigenständigen Publikation sein.

Obwohl die drei in die Erhebung einbezogenen Thüringer Direktvermarkter über mangelhafte Haltbarkeit ihrer produzierten Dauerwürste klagten, wurde bei allen drei geprüften Tiergruppen von den Schweizer Kriterien für gute Fettqualität im Rückenspeck im Mittel nur das Merkmal C18:3-Anteil überschritten (Tab. 3). Die Schweine mit einer Schlachtmasse von 90,5 kg erreichten mit 2,05 \% erwartungsgemäß den höchsten Wert. Trotz einer Schlachtmasse von 142,4 bzw. 144,2 kg war auch bei den sogenannten „Wurst“-Schweinen der Betriebe 1 und 3 der C18:3-Gehalt des Rückenfetts mit ca. 1,5\% für die Herstellung von Dauerwurst wahrscheinlich noch zu hoch. Entsprechend nahm das Verhältnis von n-6- zu n-3-PUFA Werte an, die nahezu den Empfehlungen der Deutschen Gesellschaft für Ernährung entsprachen. Aus der Analyse des eingesetzten Futters (Tab. 4) folgte, dass dessen PUFA-Gehalt mit 8,2 g/kg noch unterhalb des von BRACHER u.a. (2002) vorgeschlagenen Grenzwertes für „Salamifutter" $(9,45 \mathrm{~g} / \mathrm{kg})$ lag. Der mit $6 \%$ sehr hohe Anteil von C18:3 am Rohfett des Futters dürfte wegen der ausgeprägten Oxidationsempfindlichkeit der Linolensäure die Ursache für die Qualitätsprobleme bei den Dauerwurstwaren darstellen. NÜRNBERG u.a. (1994) erreichten in einem Mastversuch bereits bei $10 \%$ Rapskuchenanteil an der Ration C18:3-Anteile im Rückenfett von über 1,5\%, ohne dass dessen PUFA-Gehalt 10 $\%$ übertraf. SCHÖNE u.a. (2002) erzielten bei Pietrainschweinen bereits mit 7,5\% Rapspresskuchenanteil an der Ration 1,5 \% C18:3 im Rückenfett. Durch Absenkung des Anteils von Rapsverarbeitungsprodukten an der Ration konnte wieder die ge- 
wünschte Dauerwurstqualität erzielt werden. Hinsichtlich der Empfehlungen zu tolerierbaren PUFA-Anteilen im Futter und im Rückenspeck von Schweinen variieren die Literaturangaben stark von $<10 \mathrm{bis}>20 \mathrm{~g} / \mathrm{kg} \mathrm{bzw} .12-20 \%$. Eine Reihe von Autoren (vgl. SCHULZ und ALERT, 2002) gaben zwischen beiden PUFA-Merkmalen auch Regressionen an. SCHEEDER u.a. (2000) wiesen aber darauf hin, dass zwischen ihnen auch nicht-lineare Abhängigkeiten bestehen können. Da neben der Fettsäurenzusammensetzung der Ration, auch deren Vitamin E-Gehalt (FLACHOWSKY u.a., 1997), Rasse, Geschlecht, Mastgewicht sowie weitere Faktoren Konsistenz und oxidative Stabilität des Rückenfetts beeinflussen, bietet die Orientierung an den als streng geltenden Schweizer PUFA-Grenzwerten im Futter und Rückenfett eine größere Sicherheit für die Produktion von hochwertigen Dauerwaren als eine tolerantere Auffassung.

Das extrahierte Wurstfett wies bei fast allen Knackwurstproben (Tab. 5) PUFA-Anteile $<12 \%$ aber Linolensäureanteile (C18:3) von über $1 \%$ auf. WARNANTS u.a. (1998) empfahlen für die Produktion belgischer Salami weniger als $14 \%$ PUFA im Wurstfett. Die in Tabelle 5 aufgeführten Knackwürste erreichen beim Wurstfett im Mittel ein als ernährungsphysiologisch günstig zu bewertendes n-6- / n-3-Fettsäurenverhältnis von 6,4, während die Sonnenblumenölmargarine nur mit einem Quotienten von 106,9 aufwarten kann. In diesem Zusammenhang ist es unverständlich, dass von Seiten der Humanmedizin die Menschen bei der Nährmittelwahl immer noch vor den „gesättigten Fettsäuren von Schweinefett“ gewarnt werden und man zur Fettsäurenzusammensetzung bei Haustieren Literatur von 1968 zitiert (vgl. STRUNZ und JOPP, 2002). Das bei 00-Rapsöl (erucasäure- und glucosinolatarme Sorte) anzutreffende Verhältnis von C18:2 zu C18:3 von 1 : 1,35 deutet auf einen Futterbestandteil hin, der für den hohen Anteil an C18:3 im Wurstfett der Stichprobe hauptsächlich verantwortlich sein dürfte. Den damit verbundenen Haltbarkeitsproblemen tragen die industriellen Wursthersteller bereits Rechnung, indem sie Produkte wie Knackwurst oder Salami unter Schutzgasatmosphäre verpacken und ein Mindesthaltbarkeitsdatum angeben.

5. Schlussfolgerungen

Fett aus dem äußeren Rückenspeck Thüringer Schweine besitzt bei den gegenwärtigen Herkünften einen Anteil ungesättigter Fettsäuren von über $45 \%$ sowie ein günstiges Verhältnis von $\mathrm{n}-6-\mathrm{zu}$ n-3-Fettsäuren zwischen 4 und 11. Die partieweise Bestimmung der Iodzahl von Rückenfett gestattet eine repräsentative Erfassung seines durchschnittlichen Anteils an $\mathrm{C}=\mathrm{C}$-Doppelbindungen. Die durch Futterzusammensetzung, genetische Konstruktion, Geschlecht und Mastdauer bestimmte Zusammensetzung des Rückenfetts von Schweinen spiegelt sich auch im Fettsäurenmuster von Knackwurst aus dem Thüringer Einzelhandel summarisch wider. Vor allem ein Linolensäuregehalt $>1 \%$ und ein für Lipide tierischer Herkunft ernährungsphysiologisch bemerkenswert günstiger Quotient der n-6- und n-3-Fettsäuren von 6- 7 kennzeichnet derzeitig das Fett dieser Würste. Es muss gezielten Versuchen vorbehalten bleiben, zur Qualitätssicherung bei Konsistenz und Haltbarkeit für das Fett von Thüringer Dauerwurst spezifische Grenzwerte an Linolsäure, Linolensäure und der Summe von Polyenfettsäuren zu definieren. 


\section{Literatur}

BELITZ, H.-D.; GROSCH, W.:

Lehrbuch der Lebensmittelchemie. 4. Auflage, Springer-Verlag Berlin Heidelberg, 1992

BODIS, K.:

Untersuchungen zur Nahen-Infrarot (NIR)-Meßmethodik als Möglichkeit zur Schnellbestimmung der Fleisch- und Fettbeschaffenheit beim Schwein. TU München, Diss., 1999

BRACHER, A.; STOLL, P.; JOST, M.:

Abgehende Muttersauen eignen sich gut für die Salamiproduktion. AgrarForschung 9 (2002) 1, 54-59

DECKER, M.; R $\varnothing N N$, B.; J $\varnothing R G E N S E N$, S.S.:

Thermally assisted in-line methylation and gas chromatography with statistical data analysis for determination of fatty acid distribution and fingerprinting of plant seeds and oils. Eur. Food Res. Technol. 211 (2000), 366-373

DEUTSCHE GESELLSCHAFT FÜR ERNÄHRUNG:

Referenzwerte für die Nährstoffzufuhr. Frankfurt am Main, Umschau Braus GmbH, 2000

ENDER, K.; NÜRNBERG, K.; PAPSTEIN, H.-J.:

Die zwei Seiten des Fettes - eine Standortbestimmung. Arch. Tierz., Dummerstorf 40 (1997) 1, 35-45

FLACHOWSKY, G.; SCHÖNE, F.; SCHAARMANN, G.; LÜBBE, F.; BÖHME, H.:

Influence of oilseeds in combination with vitamin E supplementation in the diet on backfat quality of pigs. Anim. Feed Sci. Technol. 64 (1997), 91-100

GASSEN, H.G.; HAMMES, W.P. (Hrsg):

Handbuch Gentechnologie und Lebensmittel, 1. Ergänzungslieferung 11/1997

GLÄSER, K.R.:

Untersuchungen zur Eignung der Fettzahl als Kriterium für die Einschätzung der Fettqualität von Mastschweinen unter dem Einfluss der Fütterung und im Hinblick auf die Qualität von Verarbeitungsprodukten. ETH Zürich, Diss., 2000

GLODEK, P.:

Sicherheit in der Produktion und Ausgeglichenheit am Markt - Hauptziele der deutschen Schweinezucht 2000. Arch. Tierz., Dummerstorf 40 (1997) Sonderheft, 41-47

IUPAC 2.205:

Determination of the iodine value. In: Standard Methods for the Analysis of oils, fats and derivatives. 1992

JAHREIS, G.; BOCHMANN, K.:

Speisefette im Vergleich: Zur physiologischen Wirkung enthaltener Fettsäuren. Ernährungs-Umschau 45 (1998) 6, 192-197

KREUZER, M.:

Futterfett im Spannungsfeld von Beschaffenheit und dietätischer Qualität der Produkte. 3. Tagung Schweine- und Geflügelernährung, 29.11.-1.12.1994 in Halle/Saale,Tagungsband, 56-63

MÜLLER, S.; REICHARDT, W.; HARTUNG, H.; ECKERT, B.:

Analyse der Fettsäurenzusammensetzung des Rohfettes von Prüffutter für Schweine. Arch. Tierz., Dummerstorf 46 (2003) 3, 273-276

NÜRNBERG, K.; KRACHT, W.; NÜRNBERG, G.:

Zum Einfluß der Rapskuchenfütterung auf die Schlachtkörper- und Fettqualität beim Schwein. Züchtungskunde 66 (1994) 3, 230-241

PERDRIX, M.-F.; STOLL, P.:

Wie beeinflußt das Futter die Fettzahl der Schweine ? AgrarForschung 2 (1995) 1, 21-24

PRECHT, D.; MOLKENTIN, J.:

Recent trends in the fatty acid composition of German sunflower margarines, shortenings and cooking fats with emphasis on individual C16:1, C18:2, C18:3 and C20:1 trans isomers. Nahrung / Food 44 (2000) 4, 222-228

REICHARDT, W.; ECKERT, B.:

Methodenvergleich zur Fettextraktion aus Rückenspeck bei der Bestimmung der Fettsäurenzusammensetzung und der Iodzahl. Arch. Tierz., Dummerstorf 46 (2003) 3, 269-272

SCHEEDER, M.R.L.; BOSSI, H.; WENK, C.:

Kritische Betrachtungen zur Fettzahl-Bestimmung. AgrarForschung 6 (1999) 5, I-VIII

SCHEEDER, M.R.L.; GLÄSER, K.R.; WENK, C.:

Fleisch und Fettqualität beim Schwein - Einflüsse von Fütterung und Genetik. VDLUFA-Schriftenreihe 55 (2000) Teil 4, 68-80

SCHEEDER, M.R.L.; GLÄSER, K.R.; WENK, C.:

Einflüsse von Fütterung und Genetik auf Fleisch- und Fettqualität beim Schwein. 1. Betrachtung ausgewählter Aspekte der Qualitätsproduktion von Schweinefleisch. Fleischwirt. 81 (2001a) 5, 95-96, 9899 
2. Wirkung genetischer Faktoren und Beeinflussung durch verschiedene Futterfette. Fleischwirt. 81 (2001b) $6 ; 14,16,18$

SCHÖNE, F.; TISCHENDORF, F.; KIRCHHEIM, U.; REICHARDT, W.; BARGHOLZ, J.:

Effects of high fat rapeseed press cake on growth, carcass, meat quality and body fat composition of leaner and fatter pig crossbreeds. Animal Sci. 74 (2002), 285-297

SCHWÖRER, D.; REBSAMEN, A.; LORENZ, D.:

Selection on intramuscular fat in Swiss pig breeds and the importance of fatty tissue quality. 2nd Dummerstorf Muscle Workshop, 17th - 19th May 1995, Schriftenreihe des Forschungsinstitutes für die Biologie landwirtschaftlicher Nutztiere Dummerstorf, Heft 6, 116 - 124

SCHULZ, E.; ALERT, H.-J.:

Beiträge zur Fütterung von Schweinen mit hoher Leistung. Internationaler Kongress „Wirtschaftliche Schweineproduktion unter neuen Rahmenbedingungen“ der Sächsischen Landesanstalt für Landwirtschaft, Leipzig, 28.02.-02.03.2002, Tagungsband, 59-71

STRUNZ, U.; JOPP, A.:

Fit mit Fett. Wilhelm Heyne Verlag GmbH \& Co. KG, München, 2002

WARNANTS, N.; VanOECKEL, M.J.; BOUCQUE, C.V.:

Incorporation of Dietary Polyunsaturated Fatty Acids in Pork Tissues and its Implications for the Quality of the End Products. Meat Sci. 44 (1996) 1-2, 125-144

WARNANTS, N.; VanOECKEL, M.J.; BOUCQUE, C.V.:

Effect of Incorporation of Dietary Polyunsaturated Fatty Acids in Pork Backfat on the Quality of Salami. Meat Sci. 49 (1998) 4, 435-445

WENK, C.; PRABUCKI, A.L.:

Faktoren der Qualität von Schweinefleisch. Schweiz. Arch. Tierheilk. 132 (1990), 53-63

Eingegangen: 01.10.2002

Akzeptiert: 06.05.2003

Anschrift der Verfasser

Dr. habil. WERNER REICHARDT, Dr. ERHARD GERNAND, Dr. HORST HARTUNG, BÄRBEL ECKERT, Dr. SIMONE MÜLLER, UTA BRAUN

Thüringer Landesanstalt für Landwirtschaft

Am Rennsteig 3

D-99819 Oberellen - OT Clausberg 